\title{
Ion-Polymer Interaction Analysis: an Inversion Of NMR Spin Echo Experimental Data
}

\author{
Rita C.O. Sebastião* and João P. Braga \\ Universidade Federal de Minas Gerais, Instituto de Ciências Exatas, \\ Departamento de Química, Av. Antônio Carlos, 6627, BH-MG, 31270-901 \\ Luciano S. Virtuoso and Karla A.S.F. Vello \\ Universidade Federal de Alfenas, Centro de Ciências Exatas, Curso de Química, \\ Rua Gabriel Monteiro da Silva, 700, Alfenas-MG, 37130-000 \\ Carlos N. Pacheco \\ Princeton University, Department of Chemistry, Princeton, 08544-1009, USA
}

Luis H.M. da Silva

Universidade Federal de Viçosa (UFV), Departamento de Química Av., P.H. Rolfs, sn, Viçosa-MG, 36370-000 (Received on 20 July, 2010)

\begin{abstract}
A methodology for ion-polymer interaction estimation is discussed in the present work. This method is based on the inversion of experimental spin echo NMR data using Hopfield neural network to retrieve transverse relaxation time distributions. The adopted model systems consist of aqueous solutions of poly (ethylene oxide), molar mass 1500, 4000 and $35000 \mathrm{~g} \mathrm{~mol}^{-1}$ and sodium nitroprusside (NP) at different concentrations. Dipolar interaction is investigated in this work through the reduction in the transverse relaxation time and increase in the area under the distribution curves of the PEO protons which presented a linear correlation with the NP concentration. Neural network results were compared with the Simplex optimization procedure and experimental NMR values. The proposed methodology is robust, stable, non restrictive in relation to the system and more efficient to handle experimental data.
\end{abstract}

Keywords: Interaction ion-polymer; Ill-posed inverse problem; Neural network; Transverse relaxation time distribution.

\section{INTRODUCTION}

Nuclear Magnetic Resonance (NMR) relaxometry is a very useful tool for understanding chemical and physical phenomena in complex multiphase systems. In special, works in the literature confirm the transverse relaxation function is more sensitive to structural changes than the spin-lattice relaxation and self-diffusion measurements [13]. Chemical environment induces the formation of isochromats, a physical concept that means an infinitesimal ensemble with the same frequency. The spin echo decay data can be obtained by the Carr-Purcell-Meiboom-Gill - CPMG sequence pulse, which refocuses these magnetizations and acquires information on molecular dynamics throughout the transverse relaxation time, $T_{2}$. This important parameter can be used to indirectly measure properties of organic liquid and soft-solid samples [4].

To analyze a sample through NMR relaxometry, it is crucial to obtain a reliable deconvolution of $T_{2}$ components from the discrete and noisy decaying echo signal. This is essentially an ill-conditioned inverse problem [5-9] and needs robust mathematical treatment to be solved. Hopfield neural network, adapted to inverse problem methodology [6,7], were used in the present work to obtain $T_{2}$ distributions from spin echo NMR experimental data [10] in aqueous solutions of poly(ethylene oxide) and sodium nitroprusside.

Natural or synthetic water-soluble polymers are widely applied in technological areas such as biotechnology, pharmaceuticals and adhesives $[11,12]$. This number of applications

*Electronic address: ritacos@ufmg.br has increased considerable, and polymers are expected to be the main product demanded by industrially developed societies [13]. Nevertheless, the expansion of research needs physicochemical data of macromolecular aqueous solutions, as well as the development of models to relate experimental data with molecular interaction parameters. This is an important step for understanding more complex formulations requested by the numerous technical applications.

The establishment of a systematic and effective methodology for quantitative evaluation of ion-polymer interaction in aqueous media is proposed in this work. This methodology is not restricted to a specific system and provides a wide range of applications, such as data processing in the brain tissue for the diagnosis of multiple sclerosis [8], kinetic constants inversion from absorbance measurement [14] and data processing of scattered light [15-17].

\section{THEORETICAL BACKGROUND}

\subsection{Relaxation process in a system of two spins}

Dipole-dipole interaction consists in a spin particle I interacting with a magnetic field generated by another spin particle $S$. The Hamiltonian of this interacting pair is [18]

$$
\mathrm{H}^{\prime}=-\frac{h^{2}}{\mathrm{R}^{3}} \gamma_{\mathrm{I}} \gamma_{\mathrm{S}}[3(\mathrm{I} \cdot \mathrm{n})(\mathrm{S} \cdot \mathrm{n})-\mathrm{I} \cdot \mathrm{S}]
$$

where $\mathbf{n}$ is the unitary vector in the radial direction, $\gamma$ is the gyromagnetic ratio and $\mathrm{R}$ is the internuclear distance. This term can be considered as a perturbation in the complete Hamiltonian, $\mathbf{H}$ of a system within a magnetic field, $B_{0}$, 
along the $z$ direction. In this case, the non-perturbed Hamiltonian is composed by the motion of the particles, $\mathrm{H}_{\mathrm{M}}$, and the Zeeman energies for each spin, so that

$$
\mathrm{H}=\mathrm{H}_{\mathrm{M}}-\hbar \mathrm{B}_{0} \gamma_{\mathrm{I}} \mathrm{I}_{\mathrm{Z}}-\hbar \mathrm{B}_{0} \gamma_{\mathrm{S}} \mathrm{S}_{\mathrm{Z}}+\mathrm{H}^{\prime}
$$

For spins of value $1 / 2$, according to its magnetic moments, the longitudinal components of $\mathrm{I}_{\mathrm{Z}}$ and $\mathrm{S}_{\mathrm{Z}}$ are

$$
\begin{aligned}
& \mathrm{I}_{\mathrm{Z}}|+\rangle=+\frac{1}{2}|+\rangle \\
& \mathrm{I}_{\mathrm{Z}}|-\rangle=-\frac{1}{2}|-\rangle \\
& \mathrm{S}_{\mathrm{Z}}|+\rangle=+\frac{1}{2}|+\rangle \\
& \mathrm{S}_{\mathrm{Z}}|-\rangle=-\frac{1}{2}|-\rangle
\end{aligned}
$$

From this, it can be established four eigenstates $|+\rangle|+\rangle ;|+\rangle|-\rangle ;|-\rangle|+\rangle$ and $|-\rangle|-\rangle$ with the respective population numbers $\mathrm{N}_{++} ; \mathrm{N}_{+-} ; \mathrm{N}_{-+}$and $\mathrm{N}_{--}$. Transitions between these eigenstates are possible through the transitions probabilities $\omega_{0}, \omega_{1}, \omega_{1}^{\prime}$ and $\omega_{2}$ respectively, as represented in the diagram of Figure 1.

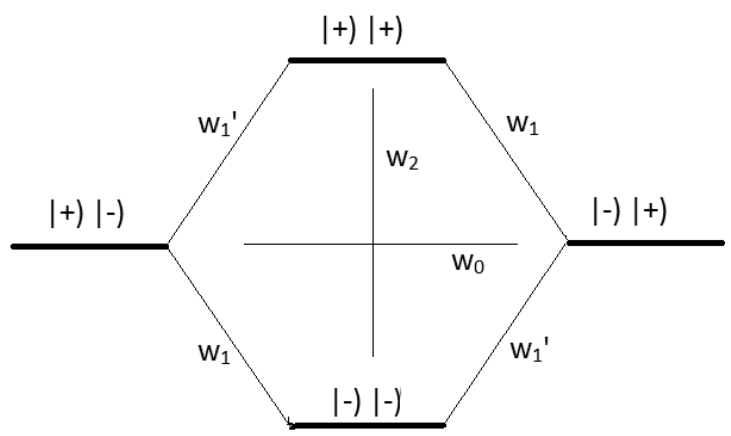

FIG. 1: Diagram of transition probabilities among the eingestates of a dipole-dipole interaction.

The longitudinal $T_{1}$ relaxation time calculation was firstly carried out by Abragam and Pound [19]. In 1955, Solomon
[18] has extended this methodology to transverse relaxation time calculation, $T_{2}$, by considering interacting pairs of like spins, nuclei in paramagnetic solution and interacting unlike spins. For this, a new basis set is defined in similarity with the equation (2) as

$$
\begin{aligned}
& \mathrm{I}_{\mathrm{x}}|\alpha\rangle=+\frac{1}{2}|\alpha\rangle \\
& \mathrm{I}_{\mathrm{x}}|\beta\rangle=-\frac{1}{2}|\beta\rangle \\
& \mathrm{S}_{\mathrm{x}}|\alpha\rangle=+\frac{1}{2}|\alpha\rangle \\
& \mathrm{S}_{\mathrm{x}}|\beta\rangle=-\frac{1}{2}|\beta\rangle
\end{aligned}
$$

In this base, four states $|\alpha\rangle|\alpha\rangle ;|\alpha\rangle|\beta\rangle ;|\beta\rangle|\alpha\rangle$ and $|\beta\rangle|\beta\rangle$ are also established, with their occupation number $\mathrm{N}_{\alpha \alpha} ; \mathrm{N}_{\alpha \beta} ; \mathrm{N}_{\beta \alpha}$ and $\mathrm{N}_{\beta \beta}$ and transition probabilities per unit of time $\mathrm{u}_{0}, \mathrm{u}_{1}, \mathrm{u}_{1}^{\prime}$ and $\mathrm{u}_{2}$, respectively. However, these states are not eigenstates of energy and the base must be defined from its orthogonal states expansion in the eigenvectors of energy, $|+\rangle$ and $|-\rangle$ as

$$
\begin{aligned}
& |\alpha\rangle=\left(\frac{1}{\sqrt{2}}\right)[|+\rangle+|-\rangle] \\
& |\beta\rangle=\left(\frac{1}{\sqrt{2}}\right)[|+\rangle-|-\rangle]
\end{aligned}
$$

Additionally, although the basis set of equation (3) can be assumed, the experimentally observable quantities are the $\mathrm{z}$-components of the macroscopic magnetizations, $\mathrm{I}_{\mathrm{z}}$ and $\mathrm{S}_{\mathrm{z}}$, being proportional to

$$
\begin{aligned}
& \left(\mathrm{N}_{++}+\mathrm{N}_{+-}\right)-\left(\mathrm{N}_{-+}+\mathrm{N}_{--}\right)=K \overline{\mathrm{I}}_{\mathrm{Z}} \\
& \left(\mathrm{N}_{++}+\mathrm{N}_{-+}\right)-\left(\mathrm{N}_{+-}+\mathrm{N}_{--}\right)=K \overline{\mathrm{S}}_{\mathrm{Z}}
\end{aligned}
$$

To develop this equation, one can consider the kinetic process of the eigenstates transition:

$$
\begin{gathered}
\frac{\mathrm{dN}_{++}}{\mathrm{dt}}=-\left(\omega_{1}+\omega_{1}^{\prime}+\omega_{2}\right) \mathrm{N}_{++}+\omega_{1}^{\prime} \mathrm{N}_{+-}+\omega_{1} \mathrm{~N}_{-+}+\omega_{2} \mathrm{~N}_{--}+\mathrm{c} \\
\frac{\mathrm{d} \mathrm{N}_{+-}}{\mathrm{dt}}=\omega_{1}^{\prime} \mathrm{N}_{++}-\left(\omega_{0}+\omega_{1}^{\prime}+\omega_{1}\right) \mathrm{N}_{+-}+\omega_{0} \mathrm{~N}_{-+}+\omega_{1} \mathrm{~N}_{--}+\mathrm{c} \\
\frac{\mathrm{d} \mathrm{N}_{-+}}{\mathrm{dt}}=\omega_{1} \mathrm{~N}_{++}+\omega_{0} \mathrm{~N}_{+-}-\left(\omega_{0}+\omega_{1}^{\prime}+\omega_{1}\right) \mathrm{N}_{-+}+\omega_{1}^{\prime} \mathrm{N}_{--}+\mathrm{c}
\end{gathered}
$$




$$
\frac{\mathrm{dN}-}{\mathrm{dt}}=\omega_{2} \mathrm{~N}_{++}+\omega_{1} \mathrm{~N}_{+-}+\omega_{1}^{\prime} \mathrm{N}_{-+}-\left(\omega_{1}+\omega_{1}^{\prime}+\omega_{2}\right) \mathrm{N}_{--}+\mathrm{c}
$$

Substituting equations (5) in the derivative form of equation (4) we get:

$$
\begin{gathered}
\frac{\mathrm{d} \overline{\mathrm{I}}_{\mathrm{Z}}}{\mathrm{dt}}=-\left(\omega_{0}+2 \omega_{1}+\omega_{2}\right)\left(\overline{\mathrm{I}}_{\mathrm{Z}}-\mathrm{I}_{0}\right)-\left(\omega_{2}-\omega_{0}\right)\left(\overline{\mathrm{S}}_{\mathrm{Z}}-\mathrm{S}_{0}\right) \\
\frac{\mathrm{d} \overline{\mathrm{S}}_{\mathrm{Z}}}{\mathrm{dt}}=-\left(\omega_{2}-\omega_{0}\right)\left(\overline{\mathrm{I}}_{\mathrm{Z}}-\mathrm{I}_{0}\right)-\left(\omega_{0}+2 \omega_{1}+\omega_{2}\right)\left(\overline{\mathrm{S}}_{\mathrm{Z}}-\mathrm{S}_{0}\right)
\end{gathered}
$$

with $I_{0}$ and $S_{0}$ being the equilibrium magnetization of spins Iand $S$.

For the transverse components, $I_{x}$ and $S_{X}$, in the basis set of equations (3), the calculation method is identical [18]. We have only to substitute the probabilities of transitions and the occupation numbers of the states, getting the equations

$$
\begin{aligned}
& \frac{\mathrm{d} \overline{\mathrm{I}}_{\mathrm{x}}}{\mathrm{dt}}=-\left(\mathrm{u}_{0}+2 \mathrm{u}_{1}+\mathrm{u}_{2}\right) \overline{\mathrm{I}}_{\mathrm{x}}-\left(\mathrm{u}_{2}-\mathrm{u}_{0}\right) \overline{\mathrm{S}}_{\mathrm{x}} \\
& \frac{\mathrm{d} \overline{\mathrm{S}}_{\mathrm{x}}}{\mathrm{dt}}=-\left(\mathrm{u}_{2}-\mathrm{u}_{0}\right) \overline{\mathrm{I}}_{\mathrm{x}}-\left(\mathrm{u}_{0}+2 \mathrm{u}_{1}+\mathrm{u}_{2}\right) \overline{\mathrm{S}}_{\mathrm{x}}
\end{aligned}
$$

In the present work, nuclei in paramagnetic solution are studied. For this case, the relaxation process of a nuclear spin, I, when paired with an electronic spin, S, of a paramagnetic ion is a consequence of the dipole-dipole interaction, $\mathrm{H}^{\prime}$. In contrast, for the electronic spin, this process is negligible and we have to consider $\overline{S_{z}}=S_{0}$ and $\overline{S_{x}}=0$, which causes a simple decay in equation (7), with the relaxation time:

$$
\frac{1}{\mathrm{~T}_{2}}=\mathrm{u}_{0}+2 \mathrm{u}_{1}+\mathrm{u}_{2}
$$

The transition probabilities are calculated by perturbation theory in first order, as in reference [18] and the transverse relaxation time of spins in paramagnetic solutions can be described by

$$
\frac{1}{T_{2}}=\frac{h^{2} \gamma_{I}^{2} \gamma_{S}^{2}}{r^{6}} \tau_{c}
$$

being $r$ the interatomic distance and $\tau c$ the rotational correlation time, or the characteristic time of a particle in solution on Brownian rotation diffusion movement.

\subsection{The spin echo theory}

The nuclear spin angular frequency, i.e. the Larmor frequency, is represented as [19,20],

$$
\omega(r)=-\gamma B(r)
$$

in which $\gamma$ is the magnetogyric ratio and $B(r)$ the local magnetic field. Due to spin-spin interactions, neighboring nuclei have different local fields and different frequencies. The spins can be refocused, forming an echo, by applying a hard $180^{\circ}$ pulse of radio-frequency after a hard $90^{\circ}$ pulse in relation to the total magnetization vector. This experiment was first verified by E.L. Hahn in 1949, after the initial discovery of NMR [20]. From this experiment, two important properties can be obtained: relaxation time and diffusion coefficient [21].

Decrease of coherence produces a magnetization decaying curve in each echo time, $t$, from which the transverse relaxation time [19-23], $T_{2}$, can be calculated. Nevertheless, distribution of $T_{2}$ components reflects the several spin-spin relaxation processes occurring inside the matter. The concept of $T_{2}$ distribution is useful since there will be a set of isochromats acting in the relaxations process. Under these theoretical considerations a single $T_{2}$ component is not possible, as in equation (9), but rather its distribution. Consequently one has to deal with multiple $T_{2}$ components, resulting in a total magnetization function [21]:

$$
I_{x y}=\sum P\left(\lambda_{i}\right) \exp \left(-t \lambda_{i}\right)
$$

with $\lambda_{i}=1 / T_{2 i}$, the decay rate for each process and $\mathrm{P}\left(\lambda_{i}\right)$ its corresponding probability.

For a continuous distribution of transverse relaxation times and the probability density function [6], $f(\lambda)=$ $P(\lambda) / d \lambda$, this equation can be written as

$$
\int K(t, \lambda) f(\lambda) d \lambda=I(t)
$$

in which $I(t)$ is the signal intensity measured at the spin echo time, $t$, and $K(t, \lambda)=\exp (-t \lambda)$ the kernel of the process. Equation (12) is a Fredholm integral equation of first order [20-22] used to calculate the relaxation time distribution function by a deconvolution process. Presence of noisy in the available data for the echo signal will make the inverse calculation of $f(\lambda)$ an inverse ill-posed problem. Therefore, one has to use robust and stable methods to solve this kind of problem. In this work, Hopfield neural network methodology was chosen $[6,7]$.

\subsection{Neural Network background}

In the neural network approach, nervous impulse simulation is performed applying a transfer function, $\phi$, in the neuron states, $u$. The activated values are propagated in the network during a learning time until a convergence criteria is reached. For this, an energy function is defined as [6]

$$
E=\frac{1}{2} \sum_{j=1}^{m}\left(P_{c a l, j}-P_{\exp , j}\right)^{2}
$$

with $P_{c a l}$ and $P_{\exp }$ the calculated and experimental properties, respectively.

Calculated spin echo decay curve, the $P_{\text {cal }}$ property, is obtained from the activated neurons, $\phi(u)$, which are changed 
TABLE 1: Sample compositions.

\begin{tabular}{cccc}
\hline Samples & Polymer $(\% \mathrm{w} / \mathrm{w})$ & $\mathrm{D}_{2} \mathrm{O}(\% \mathrm{w} / \mathrm{w})$ & $\mathrm{NP}(\% \mathrm{w} / \mathrm{w})$ \\
\hline PEO Mn 1500 & & & \\
S11 & 9.9893 & 90.0106 & 0.0000 \\
S12 & 9.9960 & 89.7473 & 0.2566 \\
S13 & 9.9877 & 89.5124 & 0.4999 \\
S14 & 9.9940 & 89.2464 & 0.7595 \\
PEO Mn 4000 & & & \\
S21 & 9.9701 & 90.0299 & 0.0000 \\
S22 & 9.9893 & 89.7544 & 0.2562 \\
S23 & 10.0090 & 89.4910 & 0.4999 \\
S24 & 10.0017 & 89.2385 & 0.7599 \\
PEO Mn 35000 & & & \\
S31 & 9.9967 & 90.0033 & 0.0000 \\
S32 & 9.9907 & 89.7563 & 0.2530 \\
S33 & 9.9887 & 89.5114 & 0.4999 \\
S34 & 10.0176 & 89.2363 & 0.7460 \\
\hline
\end{tabular}

during the learning time, $\tau$. In this case, the activated neurons in the network represent the $f(\lambda)$ curve. Thus, the derivative of the energy function is written as, [24]

$$
\frac{d E}{d \tau}=\sum_{i=1}^{n} \sum_{j=1}^{m}\left(e_{j} \frac{\partial\left(P_{c a l}\right)_{j}}{\partial \phi_{i}} \frac{\partial \phi_{i}}{\partial u_{i}} \frac{d u_{i}}{d \tau}\right)
$$

in which $n$ is the number of neurons and $e_{j}=\sum_{j=1}^{m}\left(P_{c a l, j}-\right.$ $\left.P_{\exp , j}\right)$. This is also the number of points retrieved in the transverse relaxation function $f(\lambda)$ from the inversion process of the $m$ experimental data.

The neural network convergence is assured by the error function decreasing with the learning time. For this, two conditions in the algorithm have to be imposed. The first one is establish

$$
\frac{d u_{i}}{d \tau}=-\sum_{j=1}^{m} \frac{\partial\left(P_{c a l}\right)_{j}}{\partial \phi_{i}} e_{j}
$$

transforming (14) in

$$
\frac{d E}{d \tau}=-\sum_{i=1}^{n} \frac{\partial \phi_{i}}{\partial u_{i}}\left(\frac{d u_{i}}{d \tau}\right)^{2}
$$

The transfer function as an increasing function of the neuron states, i.e. $\partial \phi / \partial u>0$, which will imply $\frac{d E}{d \tau}<0$, is the second condition to be assumed.

As the learning process is carried out, several solutions are possible to be found. Nevertheless, this neural network approach, as a consequence of the decreasing error, provides the one which best reproduces the experimental property.

Solution of Hopfield differential equations were obtained by integrating equations (15) using a fourth order RungeKutta method [25]. From an initial guess, the stopping condition of the integration process occurs if the error function reaches the minimum value, i.e. $d u_{i} / d \tau=0$.

\section{EXPERIMENTAL SECTION}

To determine experimentally the relationship between the transverse relaxation time and interaction of poly(ethylene oxide) (PEO) and sodium nitroprusside (NP) in $\mathrm{D}_{2} O$, samples containing different compositions of mixtures of these three components were prepared according to Table 1.

The poly(ethylene oxide), PEO (average molecular mass of 1500,4000 and $35000 \mathrm{~g} \mathrm{~mol}^{-1}$ ), were obtained from Sigma (USA). The Merck (Germany) salt,
$\mathrm{Na}_{2}\left[\mathrm{Fe}(\mathrm{CN})_{5}(\mathrm{NO})\right](99.0 \%)$ was of analytical grade and used without any further purification. Aqueous solutions of the polymer and nitroprusside (NP) were prepared in the concentrations of $10 \%(\mathrm{w} / \mathrm{w})$ in polymer and in the range of 0.00 to $0.75 \%(\mathrm{w} / \mathrm{w})$ in NP. The samples were kept at $20^{\circ} \mathrm{C}$ for 3 days before the measurements. The spin echo experimental data were obtained on a Bruker $400 \mathrm{MHz}$ NMR spectrometer with the CPMG sequence pulse.

\section{RESULTS AND DISCUSSION}

The assumption of transverse relaxation time distribution as a property to characterize the PEO-NP interaction is made in the present work. An specific enthalpic interaction among poly(ethylene oxide) segments and $\left[\mathrm{Fe}(\mathrm{CN})_{5} \mathrm{NO}\right]^{2-}$ anion has been demonstrated, using FTIR measurements and phase partitioning behavior in Aqueous Two Phase Systems. [26] The initial dipole-dipole interaction between the PEO protons is perturbed by salt addition. Since the NP salt contains Fe (III), the electron-nucleus dipolar interaction is a more effective mechanism, causing a reduction in relaxation time of the PEO protons, as can be seen in equation (9). Thus, changes in transverse relaxation time distributions of PEO protons in aqueous medium and ionic different concentrations were verified.

Four sets of experiments were performed with mixtures composed of polymers with different molecular weights (PEO 1500, 4000 and $35000 \mathrm{~g} \mathrm{~mol}^{-}$1) in fixed concentration of approximately $10.00 \% \mathrm{w} / \mathrm{w}$ and sodium nitroprusside at different concentrations $(0.00,0.25,0.50$ and $0.75 \% \mathrm{w} / \mathrm{w})$, as described in Table 1.

The relaxation time distribution is obtained by numerical integration of activated neuron states, equation (15), which requires an initial condition to be solved. In this work, Inverse Laplace Transform (ILT) [21] is used as initial guess for the neural network inputs. Although the ILT is used in a routine way to invert NMR echo data, its results can be considerable improved. As shown in references $[6,7,21]$ the neural network has the property of reduce the residual error in an optimization problem. Therefore, one may use the ILT as a first approximation to obtain better results, with lower residual errors. This will provide additional and more accurate results for the experimentalists.

To check the consistency of the computational method, simulated data is given to the network together with an exact initial guess; in this case activated neuron states is constant in the learning time, showing the methodology is appropriate to invert the data. Stability of the Hopfield neural network method was tested against errors in the simulated data. For errors up to $30 \%$ this approach was able to return results in fair agreement with the original data, showing the robustness of this inversion process to handle experimental error.

Using experimental spin echo decay curve, one can observes in Table 2, the maximum of $T_{2}$ distribution curves obtained from the Hopfield neural network procedure. From these results it is verified that as the concentration of NP increases, the value of $T_{2, \max }$ decreases. This can be attributed to the increased strength of ion-polymer interaction, as described in equation (9). 
TABLE 2: Transverse relaxation time and area under the distribution function for the samples.

\begin{tabular}{ccccc}
\hline Samples & $\begin{array}{c}\text { Neural } \\
\text { Network } T_{2}(\mathrm{~s})\end{array}$ & $\begin{array}{c}\text { Area under } \\
\text { peak } / 10^{-3}\end{array}$ & Simplex $\mathrm{T}_{2}(\mathrm{~s})$ & Experimental $\mathrm{T}_{2}(\mathrm{~s})$ \\
\hline S11 & 0.6095 & 1.185 & 0.5932 & 0.5937 \\
S12 & 0.5867 & 1.227 & 0.5730 & 0.5730 \\
S13 & 0.5714 & 1.270 & 0.5547 & 0.5544 \\
S14 & 0.5479 & 1.300 & 0.5417 & 0.5415 \\
& & & & \\
S21 & 0.6027 & 1.201 & 0.5839 & 0.6004 \\
S22 & 0.5867 & 1.221 & 0.5709 & 0.5716 \\
S23 & 0.5633 & 1.258 & 0.5521 & 0.5532 \\
S24 & 0.5432 & 1.313 & 0.5299 & 0.5084 \\
& & & & \\
S31 & 0.6027 & 1.187 & 0.5648 & 0.5670 \\
S32 & 0.5714 & 1.251 & 0.5620 & 0.5626 \\
S33 & 0.5469 & 1.321 & 0.5503 & 0.5506 \\
S34 & 0.5120 & 1.356 & 0.5185 & 0.5193 \\
\hline
\end{tabular}

TABLE 3: Neural network and Simplex error comparison for spin echo decay adjustment.

\begin{tabular}{rcc}
\hline & $\begin{array}{c}\text { Neural Network } \\
\text { Error }\end{array}$ & Simplex error \\
\hline PEO Mn 1500 & $2.110 \mathrm{e}-10$ & $9.303 \mathrm{e}-05$ \\
S11 & $2.829 \mathrm{e}-10$ & $9.212 \mathrm{e}-05$ \\
S12 & $7.301 \mathrm{e}-11$ & $2.264 \mathrm{e}-05$ \\
S13 & $2.880 \mathrm{e}-10$ & $8.932 \mathrm{e}-05$ \\
S14 & & \\
PEO Mn 4000 & $1.575 \mathrm{e}-10$ & $1.223 \mathrm{e}-04$ \\
S21 & $5.163 \mathrm{e}-10$ & $3.430 \mathrm{e}-04$ \\
S22 & $3.928 \mathrm{e}-10$ & $2.733 \mathrm{e}-04$ \\
S23 & $5.892 \mathrm{e}-10$ & $3.247 \mathrm{e}-04$ \\
S24 & & \\
PEO Mn 35000 & $4.085 \mathrm{e}-10$ & $3.410 \mathrm{e}-04$ \\
S31 & $1.968 \mathrm{e}-10$ & $1.255 \mathrm{e}-04$ \\
S32 & $2.673 \mathrm{e}-10$ & $1.331 \mathrm{e}-04$ \\
S33 & $8.277 \mathrm{e}-11$ & $5.053 \mathrm{e}-05$ \\
S34 & & \\
\hline
\end{tabular}

TABLE 4: Linear correlation index in: (a) Relaxation time versus NP concentration curves and (b) Area under the distribution curves versus NP concentration.

\begin{tabular}{c|c|c}
\hline Sample Set & r (a) & r (b) \\
\hline S1 & 0.9977 & 0.9962 \\
\hline S2 & 0.9970 & 0.9796 \\
\hline S3 & 0.9979 & 0.9919 \\
\hline
\end{tabular}

Distribution functions calculated by the neural network for the PEO 1500 system with $0 \%, 0.2566 \%, 0.4999 \%$ and $0.7595 \% \mathrm{NP}$ is presented in Figure 2. Peak distributions are appropriately described by a set of 32 neurons, which corresponds also the base size. Residual error is smaller if a multiple components of $T_{2}$ is adopted, as can be confirmed in Table 3. This also indicates equation (12) is appropriate to describe this problem, contrary to individual analysis of the components.

Integrals over the distributions are proportional to the number of spins in similar dynamic behavior [21,27]. In Table 2 it is also presented the area under the distributions of all systems. One can verify the area is directly proportional to NP concentration. This can be attributed to the extension of the ion-polymer interaction, once larger NP concentration affects an equally larger number of PEO protons. Figures 3 and 4 show linear correlations among NP mass concentration and $T_{2}$ parameter and the area under the distribution functions, respectively. Table 4 shows the correlation index to this assumption.

According to Brereton et.al. [2,3], the problem formu-

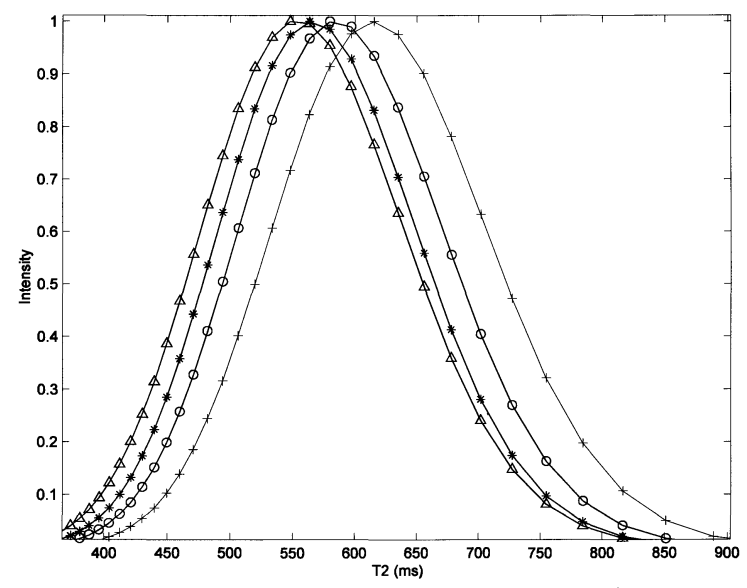

FIG. 2: Neural network transverse relaxation time distribution for PEO 1500: (+) 0\% NP, (o) $0.2566 \% \mathrm{NP},(*) 0.4999 \% \mathrm{NP}$ and $(\Delta)$ $0.7595 \%$ NP.

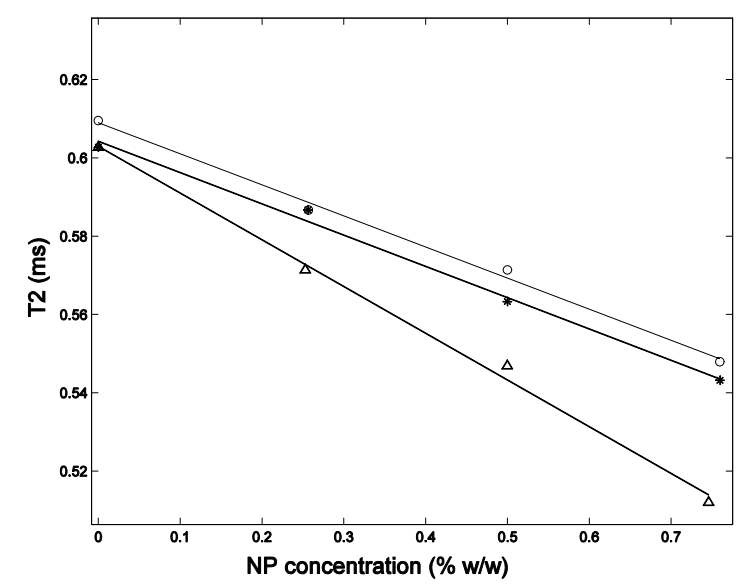

FIG. 3: Transversal relaxation time and NP concentration (\%w/w) linear correlation. The symbols are for the set of samples: (o) s1, $(*) \mathrm{s} 2$ and $(\Delta) \mathrm{s} 3$.

lation with Polyethylene melts at low molecular weights $\left(<1700 \mathrm{~g} \mathrm{~mol}^{-1}\right)$ is relatively simple since the spin echo decay curve shows a single-exponential behavior from which $T_{2}$ can be assigned. At higher molecular weights the NMR decay curve is non exponential and clearly reflects a more complex dynamics. The present study confirms that the refined treatment could reflect not only the polydispersity effects but also the differential dipolar interaction resulting from the anisotropic motion of the polymer chains on the NMR time scale.

In summary, the results presented in Table 2 and Figure 3 and 4 show two general tendencies: One is related to the direct correlation of NP concentration increase with the relaxation time decrease, which reflects an increasing strength of the interaction. The second is related to the increase in the area under the distribution curves according to the increase of the NP concentration, which reflects the larger number of spins involved in the interaction, i.e. characterize a greater 
interaction extension.

The experimental echo decay curves were also analyzed by the simplex optimization method. This method is analogue to one usually adopted by the NMR equipments, which treats the problem as an adjustment of the experimental data by one exponential curve $[28,29]$. The experimental and simplex results obtained are shown in Table 2 . At this point one can also note the better residual error results of the neural network methodology in comparison with the simplex algorithm, as presented in Table 3 .

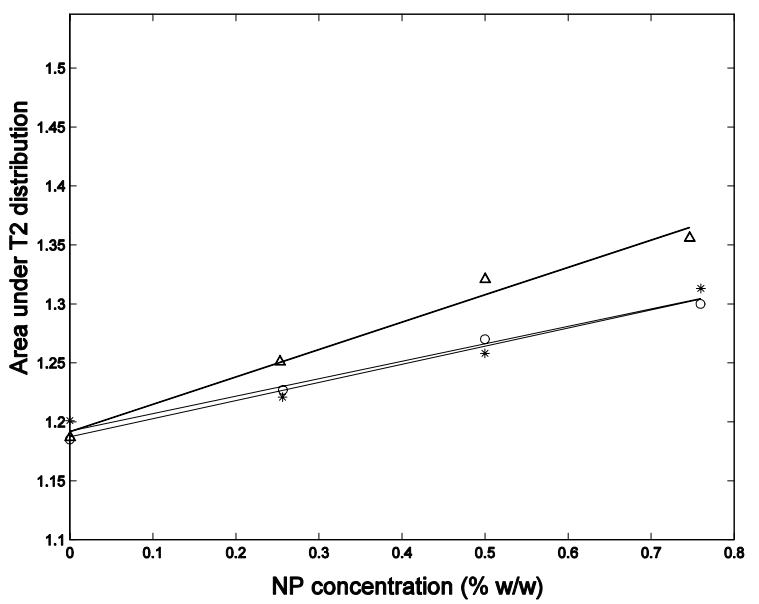

FIG. 4: Area under the $T 2$ distribution and NP concentration $(\% \mathrm{w} / \mathrm{w})$ linear correlation. The symbols are the same as in figure 2.

\section{CONCLUSION}

The combination of the inverse problem methodology and intermolecular forces analysis was applied to ion-polymer interaction estimation using experimental NMR spin echo data. The adopted model systems consist of aqueous solutions of poly (ethylene oxide) and sodium nitroprusside (NP) at different concentrations.

This inversion methodology provides $T_{2}$ distributions of the components, which reflects the several spin relaxation processes occurring inside the matter. Under these theoretical considerations, the obtained results show smaller residual errors in comparison with the simplex optimization procedure and experimental NMR equipment software. This fact is important to confirm the effectiveness of the proposed methodology.

The system responses also allow a linear correlation among the nitroprusside concentration and the transverse relaxation time, together with the PEO protons amount in the similar dynamic behavior. This linear correlation is proposed here as a quantitative approach for ion-polymer interaction estimation in aqueous solutions. Finally, the PEO molecular weight dependence in the transverse relaxation time and the area under the distribution curves shows the angular correlation is an individual characteristic of each material, as verified for the response of all sample sets.

\section{Acknowledgement}

This work was supported by PRPq, Fapemig and CNPq.
[1] S. Ghosh, K.M. Keener, Y. Pan. J. Magn. Reson. 191, 226 (2008).

[2] M. G. Brereton. Macromolecules 23, 1119 (1990).

[3] M. G. Brereton, I. M. Ward, N. Boden, P. Wright. Macromolecules 24, 2068 (1991).

[4] R.I. Chelcea,; R. Fechete, E. Culea, D.E. Demco, B.Blmich. J. Magn. Reson. 196, 178 (2009).

[5] Hadamard, J.; Le probleme de Cauchy et les equations aux derivees partielles lineaires hyperboliques, Hermann: CityplaceParis, 1932.

[6] Hopfield, J.J.; Proc. Natl. Acad. Sci. 81-10, 3088 (1984).

[7] Hopfield, J.J.; Tank, D.W.; Biol. Cybern. 52, 141 (1985).

[8] R.C.O. Sebastião, C.N. Pacheco, J.P.Braga, D.Pil-Veloso. J. Magn. Reson. 182, 22 (2006).

[9] R.C.O. Sebastião, J.P.Braga, C.N. Pacheco. Ann. Magn. Reson. 4, 1-2, 17 (2005).

[10] K.P. Withall. J. Magn. Reson. 84, 134 (1999).

[11] S. J. Huang, T. A. Seery, G. Swift. J. Macromol. Sci. Pure A 7, 36 (1999)

[12] J. H. Guo, G. W. Skinner, W. W. Harcum, P. E. Barnum. Pharm. Sci. Technol. Today 6, 254 (1998).

[13] F. Lo, J. Petchonka, J. Hanly. J. Chem. Eng. Prog. 55, 7 (1993).

[14] N.H.T. Lemes, E. Borges, J.P. Braga, Chemom. Intell. Lab. Syst. 96, 84 (2009).

[15] L.S. Virtuoso, R. C. O.Sebastião, J.P. Braga, L.H.M. Da Silva.
Int. J. Quant. Chem. 1062731 (2006).

[16] R.C.O. Sebastião, N.H.T. Lemes, L.S. Virtuoso, J.P. Braga. Chem. Phys. Lett. 378, 406 (2003).

[17] E. Borges, N.H.T.Lemes, J.P. Braga. Chem. Phys. Lett. 423, 357 (2006).

[18] I. Solomon. Phys. Rev. 99-2, 559 (1955).

[19] A. Abragam, The principles of nuclear magnetism, Oxford Press, Oxford, 2004.

[20] E.L. Hahn. Phys.Rev. 80-4, 580 (1950)

[21] R.C.O. Sebastião, J.P. Braga. J. Magn. Reson. 177-1, 146 (2005).

[22] K.P. Withall. J. Magn. Reson. 93, 221 (1991).

[23] R.J.S. Brown. J. Magn. Reson. 132, 65 (1998).

[24] Vemuri, V.; Jang, G. S.; J. Franklin Inst. 329, 241(1992).

[25] Forsythe, G.E.; Malcolm, M.A.; Moler, C.B.; Computer Methods for Mathematical Computations, Englewood Clifs: New Jersey, 1977.

[26] L.H.M. Da Silva, M.C.H. Silva, K.R. Francisco, M.V.C. Cardoso, L.A. Minim, J.S.R. Coimbra. J. Phys. Chem. B 112, 11669 (2008).

[27] V.C. Viterbo, R.C.O. Sebastião, J.P. Braga, W. F. Magalhães. J. Braz. Chem. Soc. 16, 93 (2005).

[28] Levenberg , K.; Quart. Appl. Math. 2, 164 (1944).

[29] Marquardt, D.; SIAM J. Appl. Math. 11, 431(1963). 